\title{
Depression, anxiety and stress symptoms among diabetics in Malaysia: a cross sectional study in an urban primary care setting
}

\author{
Gurpreet Kaur ${ }^{\text {** }}$ Guat Hiong Tee ${ }^{1}$, Suthahar Ariaratnam², Ambigga S Krishnapillai ${ }^{2}$ and Karuthan China ${ }^{3}$
}

\begin{abstract}
Background: Diabetes mellitus is a highly prevalent condition in Malaysia, increasing from $11.6 \%$ in 2006 to $15.2 \%$ in 2011 among individuals 18 years and above. Co-morbid depression in diabetics is associated with hyperglycemia, diabetic complications and increased health care costs. The aims of this study are to determine the prevalence and predictors of depression, anxiety and stress symptoms in Type II diabetics attending government primary care facilities in the urban area of Klang Valley, Malaysia.
\end{abstract}

Methods: The study was cross sectional in design and carried out in 12 randomly selected primary care government clinics in the Klang Valley, Malaysia. A total of 2508 eligible consenting respondents participated in the study. The Depression, Anxiety and Stress Scale (DASS) 21 questionnaire was used to measure depression, anxiety and stress symptoms. Data was analyzed using the SPSS version 16 software using both descriptive and inferential statistics.

Results: The prevalence of depression, anxiety and stress symptoms among Type II diabetics were 11.5\%, 30.5\% and $12.5 \%$ respectively. Using multiple logistic regression, females, Asian Indians, marital status (never married, divorced/ widowed/separated), a family history of psychiatric illness, less than 2 years duration of diabetes and current alcohol consumption were found to be significant predictors of depression. For anxiety, unemployment, housewives, HbA1c level of more than $8.5 \%$, a family history of psychiatric illness, life events and lack of physical activity were independent risk factors. Stress was significantly associated with females, $\mathrm{HbA1c}$ level of more than $8.5 \%$, presence of co-morbidity, a family history of psychiatric illness, life events and current alcohol consumption. For depression (adjusted OR 2.8, 95\% Cl 1.1; 7.0), anxiety (adjusted OR 2.4, 95\% Cl 1.1;5.5) and stress (adjusted OR 4.2, 95\% Cl 1.8; 9.8), a family history of psychiatric illness was the strongest predictor.

Conclusion: We found the prevalence of depression, anxiety and stress symptoms to be high among Type II diabetics, with almost a third being classified as anxious. Screening of high risk Type II diabetics for depression, anxiety and stress symptoms in the primary care setting is recommended at regular intervals.

Keywords: Depression, Anxiety, Stress, Prevalence, Predictors, Diabetes, Outpatients, Urban, Malaysia

\section{Background}

Diabetes and depression are two of the commonest public health problems affecting people all over the world. About 220 million people are estimated to be suffering from diabetes, majority of the burden being in low and middle income countries (LMIC) [1]. Diabetes is also responsible for about 1.256 million deaths globally in 2008 , with most

\footnotetext{
* Correspondence: dr.gurpreet@iku.moh.gov.my

${ }^{1}$ Institute for Public Health, Ministry of Health, Jalan Bangsar, Kuala Lumpur 50590, Malaysia

Full list of author information is available at the end of the article
}

deaths occurring in LMIC. Unipolar depressive disorders and diabetes were ranked $3^{\text {rd }}$ and $19^{\text {th }}$ respectively as leading causes of disability adjusted life years (DALYs) in 2004. The former also being the leading cause of years lost due to disability (YLD). Unipolar depressive disorders are in fact projected to be the leading cause of disease burden by 2030 .

In Malaysia, a middle income country, the prevalence of diabetes has increased in the last decade from $5.7 \%$ to 9.5\% among individuals aged 30 years and above [2] and from $11.6 \%$ in 2006 to $15.2 \%$ in 2011 among individuals

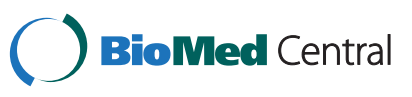


18 years and older[3]. Of the estimated 2.6 million diabetics in Malaysia, about 715,550 (27.5\%) diabetics are originating from the most populous regions in Malaysia namely the state of Selangor and the Federal Territory of Kuala Lumpur [3]. In terms of leading causes of total YLD, the Malaysian National Burden of Disease and Injury Study 2004 ranked diabetes mellitus as the third leading cause in both males (6.0\%) and females $(7.2 \%)$ respectively, while unipolar major depression was ranked as second and top most leading cause in males $(7.2 \%)$ and females $(12.7 \%)$ respectively [4].

It is well recognized that many individuals with chronic illnesses also have co-morbid unrecognized mental health disorders [5]. The International Federation of Diabetes has stressed the importance of integrating psychological care in the management of diabetes [6].

It has been estimated that the risk of getting depression in the general population is $10-25 \%$ in females and $5-12 \%$ in males. For individuals with chronic illnesses, the risk is higher at $25-33 \%$ [7]. Studies have shown that diabetics have a higher prevalence of depression than non-diabetic populations [8-10]. Globally, an estimated 43 million diabetics have symptoms of depression [5]. Also, diabetes is associated with anxiety disorders [11]. Being diagnosed with diabetes is a life stressor by itself. It requires a large number of physical and mental accommodations. Depression adds to the burden of managing diabetes. Furthermore, health care utilization and costs [12-14] increase with the coexistence of diabetes and major depression.

Depression and anxiety are associated with hyperglycemia [15-17]. While depression is associated with diabetes complications $[18,19]$ and increased functional disability [20,21]. Co-morbid depression has also been shown to be associated with poor adherence to diabetes medication and dietary regimens $[16,22,23]$ and reduced quality of life [24,25]. Several studies have shown the risk of mortality to be increased by depression [26-29]. The PROSPECT trial has shown that the five year risk of mortality was reduced with a depression management care program among diabetics compared to similar patients with usual care practices [30].

In Malaysia, there is paucity of epidemiological estimates on the prevalence, characterization and risk factors of depression, anxiety and stress among diabetics. An estimate of the prevalence of these conditions is the first step towards priority setting and the planning, implementation and evaluation of a depression management intervention program in diabetes care in the primary care setting. This study was carried out with the aims of determining the prevalence of depression, anxiety and stress, and its predictors among Type II diabetic outpatients attending government primary care clinics in the Klang Valley.

\section{Methods}

This was a cross sectional study carried out in 12 selected government primary care clinics located in the Klang Valley.

\section{Study location}

The Klang Valley generally refers to the urban areas of Kuala Lumpur, its suburbs and adjoining areas in the state of Selangor. To the North and East, it is demarcated by the Titiwangsa Mountain range, while to the West by the Straits of Malacca. The estimated population of the Klang Valley is 7.5 million [31]. The two federal territories of Kuala Lumpur and Putrajaya as well as five districts from the state of Selangor (Sepang, Hulu Langat, Gombak, Klang and Petaling) were included in the study. In each of these localities the number of clinics ranged from 1 to 13 with a total of 45 clinics.

\section{Sample size and sampling}

Sample size was calculated using both the population survey method for prevalence and for comparing two proportions using the Sample Size Calculator for Prevalence Studies [32] and the PS Software [33] respectively. The larger minimum sample size required based on both these methods was taken as the sample size for the whole study. Based on $80 \%$ study power, Type I error of 0.05 , design effect of 2 , a difference of $8 \%$ in two groups and a non-response of $20 \%$, a sample size of 2261 was required.

For sampling, a two stage stratified sampling technique was employed. About $25 \%$ of the total number of clinics from each locality (with a minimum of one clinic) was randomly selected, giving a total of 12 clinics from the study area. The sample size was then proportionately distributed based on the number of clinics selected from each locality. For three localities where only one single clinic was selected, the minimum sample size was increased to at least 250 to enable clinic level analyses in future. The final minimum sample size for the study was 2446.

\section{Study procedure}

All patients attending the diabetic clinics during the study period were screened for eligibility to participate. The inclusion criteria were age 30 years and above, having Type II diabetes of at least six months (verified with medical records) and being literate in Malay (which is the official language of the country) or English. Patients with a known medically diagnosed psychiatric illness in the past (verified with medical records) or with any form of cognitive impairment such as dementia or mental retardation and females in the post-partum period were excluded. Eligible patients were then approached for written consent for the study. Prior to obtaining consent, 
Table 1 Frequency distribution of respondents by socio-demographic characteristics and selected variables

\begin{tabular}{|c|c|c|c|c|}
\hline $\begin{array}{l}\text { Demographic characteristics } \\
(\mathrm{n}=2508)\end{array}$ & Mean & SD & $\mathrm{n}$ & $\begin{array}{c}\text { Percentage } \\
(\%)\end{array}$ \\
\hline Age (years) & 56.6 & 10.67 & & \\
\hline $30-39$ & & & 158 & 6.3 \\
\hline $40-49$ & & & 458 & 18.3 \\
\hline $50-59$ & & & 891 & 35.5 \\
\hline $60-69$ & & & 728 & 29.0 \\
\hline $70-79$ & & & 238 & 9.5 \\
\hline$\geq 80$ & & & 35 & 1.4 \\
\hline \multicolumn{5}{|l|}{ Sex } \\
\hline Male & & & 975 & 38.9 \\
\hline Female & & & 1533 & 61.1 \\
\hline \multicolumn{5}{|l|}{ Ethnicity $^{a}$} \\
\hline Malay & & & 1282 & 51.2 \\
\hline Chinese & & & 438 & 17.5 \\
\hline Asian Indian & & & 787 & 31.3 \\
\hline
\end{tabular}

\section{Highest Educational Level}

\begin{tabular}{lcc}
\hline None & 285 & 11.4 \\
\hline Never completed primary school & 423 & 16.9 \\
\hline Completed primary school & 519 & 20.7 \\
\hline $\begin{array}{l}\text { Never completed secondary } \\
\text { school }\end{array}$ & 462 & 18.4 \\
\hline Completed secondary school & 523 & 20.9 \\
\hline A-Level/STPM/HSC & 184 & 7.3 \\
\hline Tertiary & 112 & 4.5 \\
\hline
\end{tabular}

Monthly household income $\quad 1,974.6 \quad 1,869.12$

(MHI) in Malaysian Ringgit

$\left(M^{\prime} R^{\mathrm{b}}\right)$

\begin{tabular}{lcc}
\hline$<400$ & 114 & 4.5 \\
\hline $400-699$ & 277 & 11.0 \\
\hline $700-999$ & 313 & 12.5 \\
\hline $1000-1999$ & 798 & 31.8 \\
\hline $2000-2999$ & 510 & 20.3 \\
\hline $3000-3999$ & 207 & 8.3 \\
\hline $4000-4999$ & 105 & 4.2 \\
\hline$\geq 5000$ & 184 & 7.3 \\
\hline
\end{tabular}

\section{Marital Status}

\begin{tabular}{lcc}
\hline Never married & 71 & 2.8 \\
\hline Married/Cohabiting & 2048 & 81.7 \\
\hline Divorced/Separated/Widowed & 389 & 15.5 \\
\hline Current Job Status & &
\end{tabular}

\begin{tabular}{lcc}
\hline Civil servant & 227 & 9.0 \\
\hline Private Sector employee & 448 & 17.8 \\
\hline Self-employed & 171 & 6.8 \\
\hline Government retiree & 210 & 8.4 \\
\hline Private retiree & 158 & 6.3 \\
\hline
\end{tabular}

Table 1 Frequency distribution of respondents by socio-demographic characteristics and selected variables (Continued)

\begin{tabular}{|c|c|c|c|c|}
\hline Studying and working & & & 2 & 0.2 \\
\hline Student & & & 2 & 0.1 \\
\hline Housewife & & & 781 & 31.1 \\
\hline Unemployed & & & 509 & 20.3 \\
\hline BMI $\left(\mathrm{kg} / \mathrm{m}^{2}\right)$ & 27.83 & 5.395 & & \\
\hline Underweight $(<18.5)$ & & & 28 & 1.1 \\
\hline Normal $(\geq 18.5-<25.0)$ & & & 751 & 29.9 \\
\hline Overweight $(\geq 25.0-<30.0)$ & & & 1034 & 41.2 \\
\hline Obese $(\geq 30.0)$ & & & 695 & 27.7 \\
\hline
\end{tabular}

\section{Abdominal Obesity}

\begin{tabular}{|c|c|c|c|c|}
\hline No & & & 540 & 21.5 \\
\hline Yes & & & 1968 & 78.5 \\
\hline Serum Cholesterol ( $\mathrm{mmol} / \mathrm{l})$ & 5.03 & 0.878 & & \\
\hline$<5.2$ & & & 1767 & 70.5 \\
\hline$\geq 5.2$ & & & 741 & 29.5 \\
\hline $\mathrm{HbA1c}(\%)^{\mathrm{a}}$ & 8.36 & 2.038 & & \\
\hline$\leq 8.5$ & & & 1537 & 61.3 \\
\hline$>8.5$ & & & 970 & 38.7 \\
\hline Duration of Diabetes (years) & 7.7 & 6.26 & & \\
\hline$<2$ yrs & & & 206 & 8.2 \\
\hline $2-<10$ yrs & & & 1521 & 60.6 \\
\hline $10-<20$ yrs & & & 586 & 23.4 \\
\hline$\geq 20 \mathrm{yrs}$ & & & 195 & 7.8 \\
\hline \multicolumn{5}{|l|}{ Co-morbidity ${ }^{c}$} \\
\hline No & & & 530 & 21.1 \\
\hline Yes & & & 1978 & 78.9 \\
\hline \multicolumn{5}{|l|}{ Psychiatric illness in family } \\
\hline No & & & 2484 & 99.0 \\
\hline Yes & & & 24 & 1.0 \\
\hline \multicolumn{5}{|l|}{ Diabetes in family } \\
\hline No & & & 661 & 26.4 \\
\hline Yes & & & 1847 & 73.6 \\
\hline
\end{tabular}

Life events within the last 6 months

\begin{tabular}{lcc}
\hline No & 1373 & 54.7 \\
\hline Yes & 1135 & 45.3 \\
\hline Smoking status ${ }^{\dagger}$ & & \\
\hline Non-smoker & 2089 & 83.3 \\
\hline Former smoker & 160 & 6.4 \\
\hline Current smoker & 259 & 10.3 \\
\hline Alcohol Consumption Status & & \\
\hline Lifetime abstainer & 2326 & 92.7 \\
\hline Former drinker & 88 & 3.5 \\
\hline Current drinker & 94 & 3.8 \\
\hline
\end{tabular}


Table 1 Frequency distribution of respondents by socio-demographic characteristics and selected variables (Continued)

Leisure-time Physical Activity

Status§

\begin{tabular}{lcc}
\hline Regular activity & 793 & 31.6 \\
\hline Some activity & 483 & 19.3 \\
\hline Inactive & 1232 & 49.1 \\
\hline
\end{tabular}

Life events within the last 6

monthsף

\begin{tabular}{lll}
\hline No & 1373 & 54.7 \\
\hline Yes & 1135 & 45.3 \\
\hline
\end{tabular}

a one missing data.

${ }^{b_{1}}$ USD is approximately 3 MYR.

c Co-morbidity -any chronic co-morbid condition present.

${ }^{\dagger}$ Non-smoker - Respondent who reported to have never smoked at least 100 cigarettes in his lifetime.

Current smoker- Respondents who reported to have smoked 100 or more cigarettes in his lifetime and currently smoked daily or some days. Former smoker - Respondents who reported to have smoked 100 or more cigarettes in his lifetime but not smoking currently.

₹ a) Current drinker - persons who have had at least 12 drinks in their lifetime and at least one drink in the previous year.

b) Former drinker - persons who have had $\geq 12$ drinks in their lifetime, but no drinks in the past year.

c) Lifetime abstainer - had $<12$ drinks in his/her entire lifetime.

§a) Inactive - did not report any sessions of light to moderate or vigorous leisure-time physical activity of at least 10 minutes or reported they were unable to perform leisure-time physical activity.

b) Some leisure-time activity - at least 1 session of light to moderate or vigorous activity of at least 10 minutes in duration but did not meet the requirement of regular leisure-time activity.

c) Regular leisure-time activity - at least 3 sessions per week of vigorous leisure-time activity lasting at least 20 minutes or at least 5 sessions per week of light to moderate physical activity lasting at least 30 minutes or both. I Life events - any sudden change in one's life whether desirable or undesirable.

all potential respondents were explained about the purpose of the study and the relevant procedures involved. They were assured that their blood results would be notified eventually to their attending physician. Subsequently, only consenting patients were recruited in the study.

\section{Ethical issues}

Ethical approval for the study was obtained from the Medical Research Ethics Committee, Ministry of Health Malaysia (NIHSEC 08/0809/P09). Permission to conduct the study was also obtained from the State Health Directors as well as Medical and Health Officers in charge of the selected clinics prior to the study.

\section{Data collection tools and measurements}

Socio-demographic and other relevant information were collected by five trained interviewers via face-to-face interview. Depression, anxiety and stress symptoms were measured using a self-administered short version of the Depression, Anxiety and Stress Scale (DASS), i.e., DASS 21 (Additional file 1: DAS S 21). The short version has 21 items which are divided into 7 items each assessing the symptoms of depression, anxiety and stress respectively. The DASS has been shown to have high internal consistency. The validated Malay or Bahasa Malaysia version of DASS 21 was used in this study [34]. Respondents were asked to rate their experience on each symptom over the past week on a 4-point severity scale ranging from 0 (does not apply to me), to 3 (applies to me most or all of the time). Scores for each scale were later summed up and categorized as normal, mild, moderate, severe and extremely severe according to the DASS Manual [35].

The following anthropometric and blood assay measurements were also taken:

Height and weight for Body Mass Index (BMI) - Both parameters were measured twice with the patient standing bare footed. Height (to the nearest centimeter) was measured with fixed stadiometers (Seca, Vogel \& Halke, Germany) and weight (to the nearest 0.1 kilogram) was measured using an electronic floor weighing scale (Tanita HD 319 Personal Scale, Australia). BMI was classified according to the World Health Organisation guidelines [36].

Waist circumference (WC) - Measured twice using a standard tape measure as described by the National Institutes of Health (NIDDK) [37]. Abdominal obesity was defined by a waist circumference of $\geq 90 \mathrm{~cm}$ for men and $\geq 80 \mathrm{~cm}$ for women [38].

Total Serum Cholesterol (TC) - Measured using the Accutrend GCT (Roche Diagnostics, Germany) from a single finger prick.

HbA1c level - Measured using the DCA Vantage Analyser (Siemens Healthcare Diagnostics Inc, USA) from the same single finger prick.

For all the measurements except TC and HbA1c an average of two readings was taken for analyses. Variables were categories based on clinical and statistical reasoning.

\section{Pre-test and pilot study}

A pre-test was conducted on 40 Type II diabetic patients selected conveniently from a government primary care facility which was not included in the study. This was purposively done to test the study questionnaire. The pilot study was then conducted after the pre-test in two other government health facilities that were also not selected for the study. The logistics and feasibility of conducting the study were explored. Weaknesses that were identified from the pre-test and pilot study were rectified.

\section{Data management and statistical analysis}

All questionnaires were checked for completeness of response at the clinic and attempts were made to improve 
Table 2 Frequency distribution of respondents by depression status and socio-demographic characteristics and other selected variables

\begin{tabular}{|c|c|c|c|c|c|c|c|c|}
\hline \multirow[t]{2}{*}{ Variables } & \multicolumn{4}{|c|}{$\begin{array}{l}\text { Depression } \\
\text { symptoms }\end{array}$} & \multirow[t]{2}{*}{$P$} & \multirow[t]{2}{*}{ cOR } & \multicolumn{2}{|c|}{$95 \% \mathrm{Cl}$} \\
\hline & $\begin{array}{l}\text { No } \\
(n)^{+}\end{array}$ & (\%) & $\begin{array}{l}\text { Yes } \\
(n) \neq\end{array}$ & $(\%)$ & & & Lower & Upper \\
\hline Age (Years) & & & & & 0.593 & & & \\
\hline $30-39^{*}$ & 138 & 87.3 & 20 & 12.7 & - & - & - & - \\
\hline $40-49$ & 407 & 88.3 & 54 & 11.7 & 0.752 & 0.915 & 0.53 & 1.58 \\
\hline $50-59$ & 796 & 89.5 & 93 & 10.5 & 0.413 & 0.806 & 0.48 & 1.35 \\
\hline $60-69$ & 645 & 88.6 & 83 & 11.4 & 0.655 & 0.888 & 0.53 & 1.50 \\
\hline $70-79$ & 205 & 86.9 & 33 & 13.1 & 0.730 & 1.111 & 0.61 & 2.02 \\
\hline$\geq 80$ & 28 & 82.4 & 6 & 17.6 & 0.443 & 1.479 & 0.55 & 4.01 \\
\hline \multicolumn{9}{|l|}{ Sex } \\
\hline Male* & 886 & 90.9 & 89 & 9.1 & - & - & - & - \\
\hline Female & 1332 & 87.0 & 200 & 13.0 & 0.030 & 1.49 & 1.15 & 1.94 \\
\hline $\begin{array}{l}\text { Ethnicity } \\
(n=2507)\end{array}$ & & & & & 0.006 & & & \\
\hline Malay* & 1155 & 90.1 & 127 & 9.9 & & - & - & - \\
\hline Chinese & 390 & 89.0 & 48 & 11.0 & 0.529 & 1.12 & 0.79 & 1.59 \\
\hline Asian Indian & 673 & 85.5 & 114 & 14.5 & 0.002 & 1.54 & 1.18 & 2.02 \\
\hline $\begin{array}{l}\text { Educational } \\
\text { Level }\end{array}$ & & & & & 0.006 & & & \\
\hline None & 231 & 81.9 & 54 & 18.1 & 0.001 & 2.24 & 1.37 & 3.65 \\
\hline $\begin{array}{l}\text { Primary } \\
\text { education }\end{array}$ & 837 & 88.9 & 105 & 11.1 & 0.414 & 1.20 & 0.77 & 1.86 \\
\hline $\begin{array}{l}\text { Secondary } \\
\text { education }\end{array}$ & 883 & 89.6 & 102 & 10.4 & 0.655 & 1.11 & 0.71 & 1.72 \\
\hline $\begin{array}{l}\text { Tertiary } \\
\text { education* }\end{array}$ & 268 & 90.5 & 28 & 9.5 & - & - & - & - \\
\hline $\begin{array}{l}\text { Monthly } \\
\text { household } \\
\text { income (MHI) } \\
\text { in Malaysian } \\
\text { Ringgit (MYR) }\end{array}$ & & & & & 0.006 & & & \\
\hline$<1,000$ & 92 & 80.7 & 22 & 19.3 & 0.001 & 1.90 & 1.28 & 2.80 \\
\hline $1,000-<3,000$ & 238 & 85.9 & 39 & 14.1 & 0.021 & 1.54 & 1.07 & 2.23 \\
\hline$\geq 3,000^{*}$ & 276 & 88.2 & 37 & 11.8 & - & - & - & - \\
\hline Marital Status & & & & & 0.000 & & & \\
\hline Never married & 55 & 77.5 & 16 & 22.5 & 0.001 & 2.72 & 1.53 & 4.83 \\
\hline $\begin{array}{l}\text { Divorced/ } \\
\text { widowed/ } \\
\text { separated }\end{array}$ & 1850 & 90.3 & 198 & 9.7 & 0.000 & 2.23 & 1.67 & 2.99 \\
\hline Married* & 314 & 80.7 & 75 & 19.3 & - & - & - & - \\
\hline $\begin{array}{l}\text { Current Job } \\
\text { Status }\end{array}$ & & & & & 0.013 & & & \\
\hline Unemployed & 438 & 85.4 & 75 & 14.6 & 0.005 & 1.62 & 1.16 & 2.26 \\
\hline Housewives & 682 & 87.3 & 99 & 12.7 & 0.047 & 1.37 & 1.00 & 1.82 \\
\hline Retired & 334 & 90.8 & 34 & 9.2 & 0.855 & 0.96 & 0.63 & 1.46 \\
\hline Employed* & 765 & 90.4 & 81 & 9.6 & - & - & - & - \\
\hline BMI $\left(\mathrm{kg} / \mathrm{m}^{2}\right)$ & & & & & 0.207 & & & \\
\hline$<25.0^{*}$ & 686 & 88.1 & 93 & 11.9 & - & - & - & - \\
\hline$\geq 25.0$ & 928 & 89.7 & 106 & 10.3 & 0.256 & 0.84 & 0.63 & 01.13 \\
\hline
\end{tabular}

Table 2 Frequency distribution of respondents by depression status and socio-demographic characteristics and other selected variables (Continued)

\begin{tabular}{lcccccccc}
\hline$\geq 30.0$ & 605 & 87.1 & 90 & 12.9 & 0.557 & 1.10 & 0.81 & 1.50 \\
\hline $\begin{array}{l}\text { Abdominal } \\
\text { Obesity }\end{array}$ & & & & & & & & \\
\hline No * & 484 & 89.6 & 56 & 10.4 & - & - & - & - \\
\hline Yes & 1735 & 88.2 & 233 & 11.8 & 0.344 & 1.16 & 0.85 & 1.58 \\
\hline
\end{tabular}

\section{Sr. Cholesterol}

level $(\mathrm{mmol} / \mathrm{l})$

\begin{tabular}{lcccccccc}
\hline$<5.2^{*}$ & 1555 & 88.0 & 212 & 12.0 & - & - & - & - \\
\hline$\geq 5.2$ & 664 & 89.6 & 77 & 10.4 & 0.251 & 0.85 & 0.65 & 1.12 \\
\hline HbA1c level (\%) & & & & & & & & \\
\hline$\leq 8.5^{*}$ & 1369 & 89.1 & 168 & 10.9 & - & - & - & - \\
\hline$>8.5$ & 849 & 87.5 & 121 & 12.5 & 0.239 & 1.16 & 0.91 & 1.49 \\
\hline $\begin{array}{l}\text { Duration of } \\
\text { diabetes } \\
\text { (years) }\end{array}$ & & & & & & & & \\
\hline$<2$ & 173 & 84.0 & 33 & 16.0 & 0.036 & 1.53 & 1.03 & 2.26 \\
\hline$\geq 2^{*}$ & 2046 & 88.9 & 256 & 11.1 & - & - & - & - \\
\hline Co-morbidity & & & & & & & & \\
\hline No* & 466 & 87.9 & 64 & 12.1 & - & - & - & - \\
\hline Yes & 1753 & 88.6 & 225 & 11.4 & 0.654 & 0.94 & 0.70 & 1.26 \\
\hline Psychiatric & & & & & & & &
\end{tabular}

\section{Psychiatric}

illness in

family

\begin{tabular}{lcccccccc}
\hline No* & 2202 & 88.6 & 282 & 11.4 & - & - & - & - \\
\hline Yes & 17 & 70.8 & 7 & 29.2 & 0.010 & 3.22 & 1.32 & 7.82 \\
\hline $\begin{array}{l}\text { Diabetes in } \\
\text { family }\end{array}$ & & & & & & & & \\
\hline No* & 582 & 88.0 & 79 & 12.0 & - & - & - & - \\
\hline Yes & 1637 & 88.6 & 210 & 11.4 & 0.688 & 0.945 & 0.718 & 1.245 \\
\hline
\end{tabular}

Life events

within the past

6 months

\begin{tabular}{lcccccccc}
\hline No* & 1221 & 88.9 & 152 & 11.1 & - & - & - & - \\
\hline Yes & 998 & 87.9 & 137 & 12.1 & 0.435 & 1.103 & 0.863 & 1.410 \\
\hline Smoking status & & & & & 0.550 & & & \\
\hline Non smoker* & 1842 & 88.2 & 247 & 11.8 & - & - & - & - \\
\hline Former smoker & 143 & 89.4 & 17 & 10.6 & 0.304 & 0.797 & 0.517 & 1.229 \\
\hline Current smoker & 234 & 90.3 & 25 & 9.7 & 0.650 & 0.887 & 0.527 & 1.491 \\
\hline Current drinker & & & & & & & & \\
\hline No* & 2140 & 88.6 & 274 & 11.4 & - & - & - & - \\
\hline Yes & 79 & 84.0 & 15 & 16.0 & 0.173 & 1.48 & 0.84 & 2.61 \\
\hline
\end{tabular}

\section{Leisure-time}

physical

activity level

\begin{tabular}{lllllllll}
\hline Inactive & 1077 & 87.4 & 155 & 12.6 & 0.103 & 1.23 & 0.96 & 1.57 \\
\hline
\end{tabular}

\begin{tabular}{lllllllll}
\hline Active $^{*}$ & 1142 & 89.5 & 134 & 10.5 & - & - & - & -
\end{tabular}

${ }_{c} \mathrm{OR}$-Crude odds ratio.

*- Reference group.

${ }^{\dagger}$ - Scores of 0-9 (normal).

$\ddagger-$ Scores of $\geq 10$ (mild, moderate, severe, extremely severe). 
the response rate for missing items. Data was entered manually into a database and cleaned before analyses. The Statistical Package for the Social Science (SPSS) version 16 software was used for both descriptive and inferential analysis. Items that were not answered by respondents were considered as missing. Univariate statistics such as mean values, standard deviations, frequencies and proportion percentages were derived for continuous and categorical variables respectively. Bivariate and multivariate analyses were used to measure the strength of association between the variables in the study and identify predictors for the outcomes of interest respectively. All tests were two-tailed with significance defined as $\mathrm{p}<0.05$. Odds ratios (OR) along with 95\% confidence levels (CI) were derived where appropriate.

\section{Results}

Out of 2774 eligible patients approached, 2508 subjects were successfully recruited, giving a response rate of $90.4 \%$.

\section{Socio-demographic, clinical and other characteristics of sample population}

Majority of the respondents were between 50 to 59 years old (35.5\%), females (61.1\%), Malays (51.2\%) and married (81.7\%). Almost $40 \%$ had some form of secondary education and one third earned a monthly household income (MHI) of MYR 1,000 to 1,999. Slightly over one third of respondents were gainfully employed. The mean age and MHI was about 57 (56.6 \pm SD 10.67) years and MYR $2,000(1,974.6 \pm$ SD 1,869.12) respectively (Table 1$)$.

Over $41 \%$ of the respondents were overweight while almost twice the proportion had abdominal obesity. Almost $30 \%$ and $40 \%$ of the respondents had an elevated TC level of $>5.2 \mathrm{mmol}$ and $\mathrm{HbA} 1 \mathrm{c}$ of $>8.5 \%$ respectively. Majority (61\%) were diagnosed as having diabetes for 2 - <10 years (Table 1 ).

Almost $80 \%$ reported having at least one co-morbid condition. Majority reported having at least one family member with diabetes $(73.6 \%)$ while only $1 \%$ reported having a history of mental illness in the family. As for life events, about $45 \%$ reported experiencing at least one life event in the past 6 months. Current smokers and current alcohol drinkers comprised $10 \%$ and $4 \%$ of the respondents respectively. Almost half of the respondents were classified as physically inactive (49.1\%).

\section{Depression, anxiety and stress}

Overall, the prevalence of depression, anxiety and stress symptoms were $11.5 \%, 30.5 \%$ and $12.5 \%$ respectively. On bivariate analysis using binary logistic regression, depression was found to be significantly associated with sex, ethnicity, educational level, MHI, marital status, current job status, duration of diabetes and family history of psychiatric illness (Table 2).
For anxiety, all socio-demographic variables; females, Asian Indian, no formal education, MHI of MYR < 3,000, divorcees/widowers, housewives and unemployment were significant. In addition, HbA1c $>8.5 \%, \geq 2$ years duration of diabetes, presence of psychiatric illness in the family, life events and physical inactivity were also significantly associated (Table 3).

Stress was significantly associated with sex, HbA1c, comorbidity, diabetes in the family, psychiatric illness in the family, life events and alcohol consumption (Table 4).

Variables with a $\mathrm{p}$ value of $<0.25$ in the bivariate analyses and thought to be important risk factors of depression, anxiety and stress were entered into the multivariate model. The forward likelihood ratio (LR) method was used to predict the associated variables for depression, anxiety and stress symptoms in three separate models. The presence of interaction between the explanatory variables was assessed prior to determining the final model.

In the final model six variables i.e., sex, ethnicity, marital status, duration of diabetes, psychiatric illness in the family and alcohol consumption were found to be predictors of depression (Table 5). The strongest predictor was psychiatric illness history with an adjusted odds ratio (aOR) of 2.8 times followed by marital status (aOR 2.5-2.1) and current alcohol consumption (aOR 1.8). Individuals with diabetes of less than two years duration were 1.6 times more likely to have depressive symptoms than individuals with diabetes of longer duration while females and Asian Indians were 1.4 times more likely to have depressive symptoms compared to males and Malay diabetic individuals.

In terms of anxiety (Table 5), age group, job status, HbA1c, family history of psychiatric illness, life events and leisure-time physical activity remained significant in the final model, while for stress five variables namely sex, HbA1c, co-morbidity, psychiatric illness in the family, life events and alcohol consumption were found to be significant contributors. Individuals with a family history of psychiatric illness were 2.4 times more likely to report anxiety. Anxiety was 1.4 times more likely in respondents experiencing life events, those physically inactive and with an $\mathrm{HbA1c}$ level of $>8.5 \%$. All diabetics who were not gainfully employed were 1.2-1.6 times more likely of experiencing anxiety.

Psychiatric illness in the family was the strongest predictor of stress having aOR of 4.2 followed by current alcohol drinkers with an aOR of 2.2. Having a highly undesirable level of HbA1c was associated with at least 1.6 times the odds of stress. Female diabetics and those with co-morbidity were 1.4 times more likely to report stress after controlling for confounders.

There was also significant correlation $(\mathrm{p}<0.01)$ between depression, anxiety and stress symptoms $(r=0.360$ for 
Table 3 Frequency distribution of respondents by anxiety status and socio-demographic characteristics and other selected variables

\begin{tabular}{|c|c|c|c|c|c|c|c|c|}
\hline \multirow[t]{2}{*}{ Variables } & \multicolumn{4}{|c|}{ Anxiety symptoms } & \multirow[t]{2}{*}{$P$} & \multirow[t]{2}{*}{ cOR } & \multicolumn{2}{|c|}{$95 \% \mathrm{Cl}$} \\
\hline & $\begin{array}{l}\mathrm{No} \\
(n)^{+}\end{array}$ & $(\%)$ & $\begin{array}{l}\text { Yes } \\
\text { (n) } \neq\end{array}$ & $(\%)$ & & & Lower & Upper \\
\hline Age (Years) & & & & & 0.006 & & & \\
\hline $30-39^{*}$ & 102 & 64.6 & 56 & 35.4 & - & - & - & - \\
\hline $40-49$ & 341 & 74.2 & 119 & 25.8 & 0.021 & 0.63 & 0.43 & 0.93 \\
\hline $50-59$ & 628 & 70.6 & 261 & 29.4 & 0.126 & 0.76 & 0.53 & 1.08 \\
\hline $60-69$ & 504 & 69.2 & 224 & 30.8 & 0.253 & 0.81 & 0.56 & 1.16 \\
\hline $70-79$ & 144 & 60.5 & 94 & 39.5 & 0.416 & 1.19 & 0.78 & 1.80 \\
\hline$\geq 80$ & 22 & 64.7 & 12 & 35.3 & 0.987 & 0.99 & 0.46 & 2.16 \\
\hline \multicolumn{9}{|l|}{$\overline{\text { Sex }}$} \\
\hline Male* $^{*}$ & 710 & 72.8 & 265 & 27.2 & - & - & - & - \\
\hline Female & 1032 & 67.3 & 501 & 32.7 & 0.004 & 1.30 & 1.09 & 1.55 \\
\hline Ethnicity & & & & & 0.022 & & & \\
\hline Malay* & 911 & 71.0 & 372 & 29.0 & - & - & - & - \\
\hline Chinese & 314 & 71.7 & 124 & 28.3 & 0.778 & 0.97 & 0.76 & 1.23 \\
\hline Asian Indian & 516 & 65.7 & 270 & 34.3 & 0.012 & 1.28 & 1.06 & 1.55 \\
\hline $\begin{array}{l}\text { Educational } \\
\text { Level }\end{array}$ & & & & & 0.070 & & & \\
\hline None & 184 & 64.6 & 101 & 35.4 & 0.045 & 1.43 & 1.01 & 2.04 \\
\hline $\begin{array}{l}\text { Primary } \\
\text { education }\end{array}$ & 629 & 66.8 & 313 & 33.2 & 0.076 & 1.30 & 0.97 & 1.73 \\
\hline $\begin{array}{l}\text { Secondary } \\
\text { education }\end{array}$ & 715 & 72.6 & 270 & 27.4 & 0.922 & 0.99 & 0.74 & 1.32 \\
\hline $\begin{array}{l}\text { Tertiary } \\
\text { education* }\end{array}$ & 214 & 72.3 & 82 & 27.7 & - & - & - & - \\
\hline $\begin{array}{l}\text { Monthly } \\
\text { household } \\
\text { income (MHI) } \\
\text { in Malaysian } \\
\text { Ringgit (MYR) }\end{array}$ & & & & & 0.001 & & & \\
\hline$<1,000$ & 457 & 64.9 & 247 & 35.1 & 0.000 & 1.64 & 1.27 & 2.12 \\
\hline $1,000-<3,000$ & 912 & 69.7 & 396 & 30.3 & 0.022 & 1.32 & 1.04 & 1.67 \\
\hline$\geq 3,000^{*}$ & 373 & 75.2 & 123 & 24.8 & - & - & - & - \\
\hline Marital Status & & & & & 0.010 & & & \\
\hline Never married & 52 & 73.2 & 19 & 26.8 & 0.626 & 0.88 & 0.51 & 1.49 \\
\hline $\begin{array}{l}\text { Divorced/ } \\
\text { widowed/ } \\
\text { separated }\end{array}$ & 1445 & 70.6 & 603 & 29.4 & 0.003 & 1.41 & 1.12 & 1.77 \\
\hline Married* & 245 & 63.0 & 144 & 37.0 & - & - & - & - \\
\hline $\begin{array}{l}\text { Current Job } \\
\text { Status }\end{array}$ & & & & & 0.000 & & & \\
\hline Unemployed & 315 & 61.4 & 198 & 38.6 & 0.000 & 1.86 & 1.47 & 2.35 \\
\hline Housewives & 530 & 67.9 & 251 & 32.1 & 0.002 & 1.40 & 1.13 & 1.74 \\
\hline Retired & 265 & 72.0 & 103 & 28.0 & 0.326 & 1.15 & 0.87 & 1.51 \\
\hline Employed* $^{*}$ & 632 & 74.7 & 214 & 25.3 & - & - & - & - \\
\hline BMI $\left(\mathrm{kg} / \mathrm{m}^{2}\right)$ & & & & & 0.472 & & & \\
\hline$<25.0^{*}$ & 529 & 67.9 & 250 & 32.1 & - & - & - & - \\
\hline$\geq 25.0$ & 721 & 69.7 & 313 & 30.3 & 0.407 & 0.92 & 0.75 & 1.12 \\
\hline$\geq 30.0$ & 492 & 70.8 & 203 & 29.2 & 0.023 & 0.87 & 0.70 & 1.09 \\
\hline
\end{tabular}

Table 3 Frequency distribution of respondents by anxiety status and socio-demographic characteristics and other selected variables (Continued)

\section{Abdominal}

Obesity

\begin{tabular}{llllllllc}
\hline No* $^{*}$ & 373 & 69.1 & 167 & 30.9 & - & - & - & - \\
\hline Yes & 1369 & 69.6 & 599 & 30.4 & 0.827 & 0.98 & 0.80 & 1.20
\end{tabular}

\section{Sr. Cholesterol}

level $(\mathrm{mmol} / \mathrm{l})$

\begin{tabular}{lcccccccc}
\hline$<5.2^{*}$ & 1215 & 68.8 & 552 & 38.2 & 0.242 & 0.90 & 0.74 & 1.08 \\
\hline$\geq 5.2$ & 527 & 71.1 & 214 & 28.9 & - & - & - & - \\
\hline $\begin{array}{l}\text { HbA1c } \\
\text { level (\%) }\end{array}$ & & & & & & & & \\
\hline$\leq 8.5^{*}$ & 1101 & 71.6 & 436 & 28.4 & - & - & - & - \\
\hline$>8.5$ & 641 & 66.1 & 329 & 33.9 & 0.003 & 1.30 & 1.09 & 1.54 \\
\hline $\begin{array}{l}\text { Duration of } \\
\text { DM (years) }\end{array}$ & & & & & & & & \\
\hline$<2$ & 158 & 76.7 & 48 & 23.3 & 0.019 & 1.49 & 1.07 & 2.09 \\
\hline$\geq 2^{*}$ & 1584 & 68.8 & 718 & 31.2 & - & - & - & - \\
\hline Co-morbidity & & & & & & & & \\
\hline No* & 382 & 72.1 & 148 & 27.9 & - & - & - & - \\
\hline Yes & 1360 & 68.8 & 618 & 31.2 & 0.141 & 1.17 & 0.95 & 1.45 \\
\hline Psychiatric & & & & & & & &
\end{tabular}

\section{Psychiatric}

illness in

family

\begin{tabular}{lcccccccc}
\hline No* & 1730 & 69.6 & 754 & 30.4 & 0.043 & 2.29 & 1.03 & 5.13 \\
\hline Yes & 12 & 50.0 & 12 & 50.0 & - & - & - & - \\
\hline
\end{tabular}

\section{Diabetes in}

family

\begin{tabular}{|c|c|c|c|c|c|c|c|c|}
\hline $\mathrm{No}^{*}$ & 477 & 72.2 & 184 & 37.8 & 0.079 & 1.19 & 0.98 & 1.45 \\
\hline Yes & 1265 & 68.5 & 582 & 31.5 & - & - & - & - \\
\hline \multicolumn{9}{|l|}{$\begin{array}{l}\text { Life events } \\
\text { within the } \\
\text { past } 6 \text { months }\end{array}$} \\
\hline $\mathrm{No}^{*}$ & 996 & 72.5 & 377 & 27.5 & - & - & - & - \\
\hline Yes & 746 & 65.7 & 389 & 34.3 & 0.000 & 1.38 & 1.16 & 1.63 \\
\hline Smoking status & & & & & 0.064 & & & \\
\hline Non smoker* & 1433 & 68.6 & 656 & 31.4 & - & - & - & - \\
\hline Former smoker & 186 & 71.8 & 73 & 28.2 & 0.030 & 0.66 & 0.45 & 0.96 \\
\hline Current smoker & 123 & 76.9 & 37 & 23.1 & 0.292 & 0.86 & 0.64 & 1.14 \\
\hline \multicolumn{9}{|l|}{ Current drinker } \\
\hline $\mathrm{No}^{*}$ & 1677 & 69.5 & 737 & 30.5 & - & - & - & - \\
\hline Yes & 65 & 69.1 & 29 & 30.9 & 0.947 & 1.015 & 0.650 & 1.59 \\
\hline
\end{tabular}

\section{Leisure-time}

physical

activity level

\begin{tabular}{lllllllll}
\hline Active* $^{*}$ & 931 & 73.0 & 345 & 27.0 & - & - & - & - \\
\hline Inactive & 811 & 65.8 & 421 & 34.2 & 0.000 & 1.40 & 1.18 & 1.66 \\
\hline
\end{tabular}

cOR -Crude odds ratio.

*- Reference group.

${ }^{\dagger}$ - Scores of 0-7 (normal).

$\ddagger-$ Scores of $\geq 8$ (mild, moderate, severe, extremely severe). 
Table 4 Frequency distribution of respondents by stress status and socio-demographic characteristics and other selected variables

\begin{tabular}{|c|c|c|c|c|c|c|c|c|}
\hline \multirow[t]{2}{*}{ Variables } & \multicolumn{4}{|c|}{ Stress symptoms } & \multirow[t]{2}{*}{$P$} & \multirow[t]{2}{*}{ cOR } & \multicolumn{2}{|c|}{$95 \% \mathrm{Cl}$} \\
\hline & $\begin{array}{l}\mathrm{No} \\
(n)^{+}\end{array}$ & $(\%)$ & $\begin{array}{l}\text { Yes } \\
\text { (n) } \neq\end{array}$ & $(\%)$ & & & Lower & Upper \\
\hline Age (Years) & & & & & 0.336 & & & \\
\hline $30-39^{*}$ & 133 & 84.2 & 25 & 15.8 & - & - & - & - \\
\hline $40-49$ & 408 & 88.5 & 53 & 11.5 & 0.159 & 0.691 & 0.413 & 1.156 \\
\hline $50-59$ & 784 & 88.2 & 105 & 11.8 & 0.160 & 0.713 & 0.444 & 1.144 \\
\hline $60-69$ & 627 & 86.1 & 101 & 13.9 & 0.525 & 0.857 & 0.532 & 1.380 \\
\hline $70-79$ & 214 & 89.9 & 24 & 10.1 & 0.092 & 0.597 & 0.327 & 1.088 \\
\hline$\geq 80$ & 28 & 82.4 & 6 & 17.6 & 0.793 & 1.140 & 0.428 & 3.037 \\
\hline \multicolumn{9}{|l|}{ Sex } \\
\hline Male* & 870 & 89.2 & 105 & 10.8 & - & - & - & - \\
\hline Female & 1324 & 86.4 & 209 & 13.6 & 0.035 & 1.31 & 1.02 & 1.68 \\
\hline Ethnicity & & & & & 0.077 & & & \\
\hline Malay* & 1135 & 88.5 & 147 & 11.5 & - & - & - & - \\
\hline Chinese & 387 & 88.4 & 51 & 11.6 & 0.920 & 1.02 & 0.73 & 1.43 \\
\hline Asian Indian & 671 & 85.3 & 116 & 14.7 & 0.030 & 1.34 & 1.03 & 1.73 \\
\hline $\begin{array}{l}\text { Educational } \\
\text { Level }\end{array}$ & & & & & 0.219 & & & \\
\hline None & 253 & 88.8 & 32 & 11.2 & 0.484 & 1.211 & 0.709 & 2.068 \\
\hline $\begin{array}{l}\text { Primary } \\
\text { education }\end{array}$ & 824 & 87.5 & 118 & 12.5 & 0.155 & 1.371 & 0.888 & 2.116 \\
\hline $\begin{array}{l}\text { Secondary } \\
\text { education }\end{array}$ & 849 & 86.2 & 136 & 13.8 & 0.051 & 1.533 & 0.998 & 2.355 \\
\hline $\begin{array}{l}\text { Tertiary } \\
\text { education* }\end{array}$ & 268 & 90.5 & 28 & 9.5 & - & - & - & - \\
\hline $\begin{array}{l}\text { Monthly } \\
\text { household } \\
\text { income (MHI) } \\
\text { in Malaysian } \\
\text { Ringgit (MYR) }\end{array}$ & & & & & 0.088 & & & \\
\hline$<1,000$ & 600 & 85.2 & 104 & 14.8 & 0.051 & 1.419 & 1.00 & 2.02 \\
\hline $1,000-<3,000$ & 1152 & 88.1 & 156 & 11.9 & 0.539 & 1.108 & 0.80 & 1.54 \\
\hline$\geq 3,000^{*}$ & 442 & 89.1 & 54 & 10.9 & - & - & - & - \\
\hline Marital Status & & & & & 0.573 & & & \\
\hline Never married & 62 & 87.3 & 9 & 12.7 & 0.906 & 1.04 & 0.51 & 2.13 \\
\hline $\begin{array}{l}\text { Divorced/ } \\
\text { widowed/ } \\
\text { separated }\end{array}$ & 1798 & 87.8 & 250 & 12.2 & 0.292 & 1.18 & 0.87 & 1.62 \\
\hline Married * & 334 & 85.9 & 55 & 14.1 & - & - & - & - \\
\hline $\begin{array}{l}\text { Current Job } \\
\text { Status }\end{array}$ & & & & & 0.152 & & & \\
\hline Unemployed & 436 & 85.0 & 77 & 15.0 & 0.043 & 1.40 & 1.01 & 1.93 \\
\hline Housewives & 679 & 86.9 & 102 & 13.1 & 0.258 & 1.19 & 0.88 & 1.60 \\
\hline Retired & 328 & 89.1 & 40 & 10.9 & 0.855 & 0.96 & 0.65 & 1.43 \\
\hline Employed* & 751 & 88.8 & 95 & 11.2 & - & - & - & - \\
\hline BMI $\left(\mathrm{kg} / \mathrm{m}^{2}\right)$ & & & & & 0.440 & & & \\
\hline$<25.0^{*}$ & 676 & 86.8 & 103 & 13.2 & - & - & - & - \\
\hline$\geq 25.0$ & 915 & 88.5 & 119 & 11.5 & 0.271 & 0.85 & 0.64 & 1.13 \\
\hline$\geq 30.0$ & 603 & 86.8 & 92 & 13.2 & 0.993 & 1.00 & 0.74 & 1.35 \\
\hline
\end{tabular}

Table 4 Frequency distribution of respondents by stress status and socio-demographic characteristics and other selected variables (Continued)

Abdominal

Obesity

\begin{tabular}{lcccccccc}
\hline No* $^{*}$ & 475 & 88.0 & 65 & 12.0 & - & - & - & - \\
\hline Yes & 1719 & 87.3 & 249 & 12.7 & 0.70 & 1.06 & 0.79 & 1.42
\end{tabular}

\section{Sr. Cholesterol}

level $(\mathrm{mmol} / \mathrm{l})$

\begin{tabular}{|c|c|c|c|c|c|c|c|c|}
\hline$<5.2^{*}$ & 1545 & 87.4 & 222 & 12.6 & - & - & - & - \\
\hline$\geq 5.2$ & 649 & 87.6 & 92 & 12.4 & 0.919 & 0.99 & 0.76 & 1.28 \\
\hline \multicolumn{9}{|c|}{$\begin{array}{l}\text { HbA1c } \\
\text { level (\%) }\end{array}$} \\
\hline$\leq 8.5^{*}$ & 1376 & 89.5 & 161 & 10.5 & - & - & - & - \\
\hline$>8.5$ & 817 & 84.2 & 153 & 15.8 & 0.000 & 1.60 & 1.26 & 2.03 \\
\hline \multicolumn{9}{|c|}{$\begin{array}{l}\begin{array}{l}\text { Duration of } \\
\text { Diabetes } \\
\text { (years) }\end{array} \\
\end{array}$} \\
\hline$<2$ & 182 & 88.3 & 24 & 11.7 & - & - & - & - \\
\hline$\geq 2^{*}$ & 2012 & 87.4 & 290 & 12.6 & 0.694 & 0.915 & 0.59 & 1.43 \\
\hline \multicolumn{9}{|c|}{ Co-morbidity } \\
\hline $\mathrm{No}^{*}$ & 477 & 90.0 & 53 & 10.0 & - & - & - & - \\
\hline Yes & 1717 & 86.8 & 261 & 13.2 & 0.049 & 1.37 & 1.00 & 1.87 \\
\hline
\end{tabular}

\section{Psychiatric}

illness in

family

\begin{tabular}{lcccccccc}
\hline No* & 2179 & 87.7 & 305 & 12.3 & - & - & - & - \\
\hline Yes & 15 & 62.5 & 9 & 37.5 & 0.001 & 4.28 & 1.86 & 9.88 \\
\hline
\end{tabular}

Diabetes in

family

\begin{tabular}{lcccccccc}
\hline No* $^{*}$ & 594 & 89.9 & 67 & 10.1 & - & - & - & - \\
\hline Yes & 1600 & 86.6 & 247 & 13.4 & 0.031 & 1.37 & 1.04 & 1.82 \\
\hline
\end{tabular}

Life events

within the

past 6 months

\begin{tabular}{lcccccccc}
\hline No* & 1227 & 89.4 & 146 & 10.6 & - & - & - & - \\
\hline Yes & 967 & 85.2 & 168 & 14.8 & 0.002 & 1.46 & 1.15 & 1.85 \\
\hline Smoking status & & & & & 0.836 & & & \\
\hline Non smoker* & 1824 & 87.3 & 265 & 12.7 & - & - & - & - \\
\hline Former smoker & 142 & 88.8 & 18 & 11.2 & 0.743 & 0.94 & 0.63 & 1.39 \\
\hline Current smoker & 228 & 88.0 & 31 & 12.0 & 0.598 & 0.87 & 0.53 & 1.45 \\
\hline Current drinker & & & & & & & & \\
\hline No* & 2119 & 87.8 & 295 & 12.2 & - & - & - & - \\
\hline Yes & 75 & 79.8 & 19 & 20.2 & 0.023 & 1.82 & 1.08 & 3.05 \\
\hline
\end{tabular}

Leisure-time

physical

activity level

\begin{tabular}{|c|c|c|c|c|c|c|c|c|}
\hline Active* & 1112 & 87.1 & 164 & 12.9 & - & - & - & - \\
\hline Inactive & 1082 & 87.8 & 150 & 12.2 & 0.608 & 0.94 & 0.74 & 1.19 \\
\hline
\end{tabular}


Table 5 Multiple logistic regression model predicting depression, anxiety and stress symptoms among type II diabetic outpatients

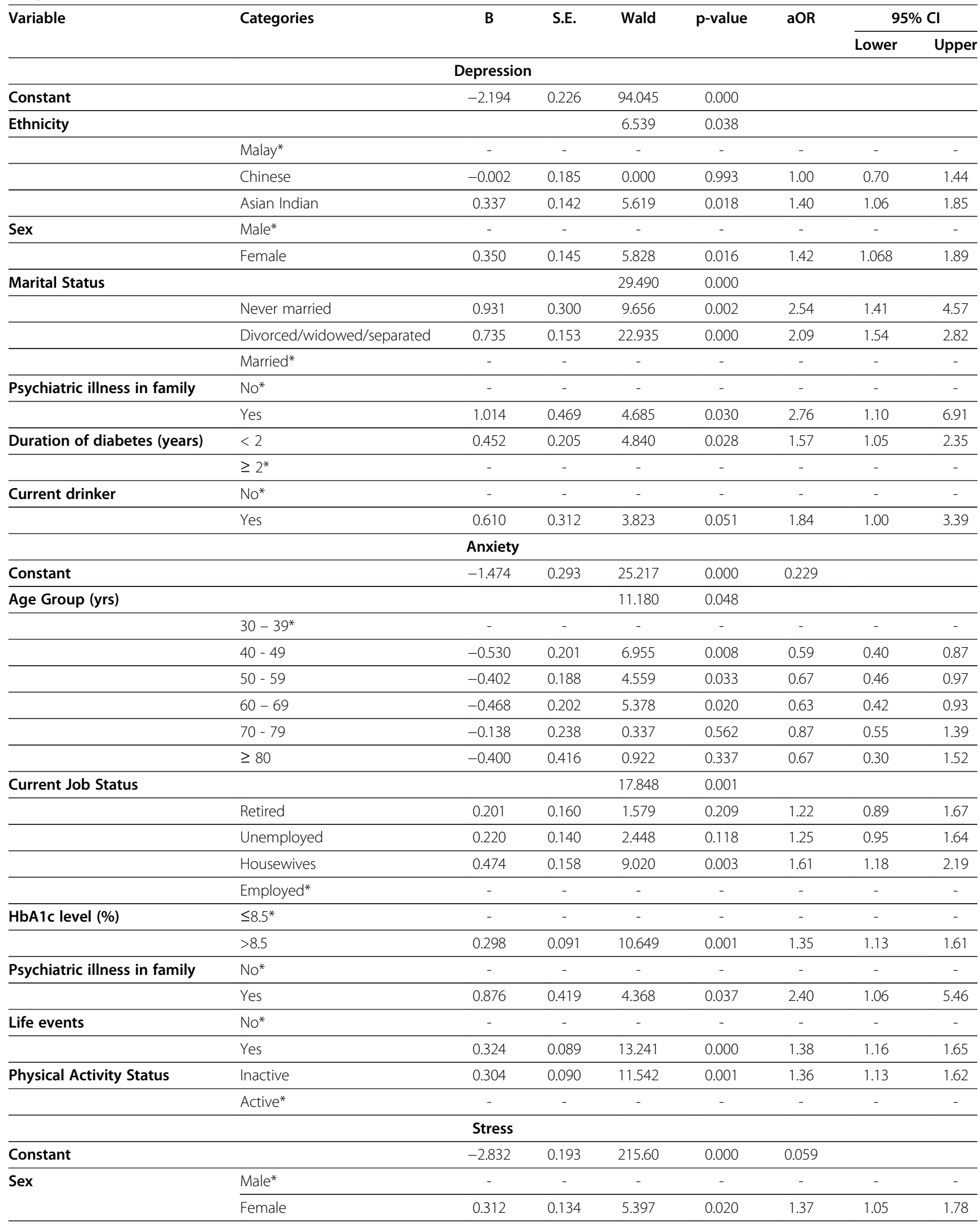


Table 5 Multiple logistic regression model predicting depression, anxiety and stress symptoms among type II diabetic outpatients (Continued)

\begin{tabular}{|c|c|c|c|c|c|c|c|c|}
\hline \multirow[t]{2}{*}{ HbA1c level (\%) } & $\leq 8.5^{*}$ & - & - & - & - & - & - & - \\
\hline & $>8.5$ & 0.493 & 0.123 & 16.119 & 0.000 & 1.64 & 1.29 & 2.08 \\
\hline \multirow[t]{2}{*}{ Co-morbidity } & $\mathrm{No}^{*}$ & - & - & - & - & - & - & - \\
\hline & Yes & 0.345 & 0.161 & 4.561 & 0.033 & 1.41 & 1.029 & 1.94 \\
\hline \multirow[t]{2}{*}{ Psychiatric illness in family } & $\mathrm{No}^{*}$ & - & - & - & - & - & - & - \\
\hline & Yes & 1.435 & .434 & 10.931 & 0.001 & 4.20 & 1.79 & 9.83 \\
\hline \multirow[t]{2}{*}{ Life events } & $\mathrm{No}^{*}$ & - & - & - & - & - & - & - \\
\hline & Yes & 0.292 & 0.123 & 5.592 & 0.018 & 1.34 & 1.05 & 1.71 \\
\hline \multirow[t]{2}{*}{ Current drinker } & $\mathrm{No}^{*}$ & - & - & - & - & - & - & - \\
\hline & Yes & 0.775 & 0.282 & 7.580 & 0.006 & 2.17 & 1.25 & 3.77 \\
\hline
\end{tabular}

aOR -adjusted odds ratio.

*-Reference group.

depression and anxiety, $\mathrm{r}=0.547$ for depression and stress and $\mathrm{r}=0.504$ for anxiety and stress).

\section{Discussion}

\section{Depression, anxiety and stress}

This study showed that the prevalence of depression, anxiety and stress symptoms were 11.5\%, 30.5\% and $12.5 \%$ respectively among Type II Diabetic outpatients in the Klang Valley, Malaysia. The prevalence of anxiety in this study was almost three fold more than that of depression and this is in keeping with current literature in which anxiety rates are frequently higher than depression [39-42].

Our findings concur with other chronic disease models such as chronic obstructive pulmonary disease (COPD), whereby a study by Dahlen \& Janson found that $12 \%$ and $37 \%$ of respondents with asthma and COPD had probable depression and anxiety respectively [43].

The depressive symptom rates we found are also comparable to studies in rural America (15.8\%) [44], the UAE (12.5\%) [45] and Germany (10.2\%) [11]. Conversely, several studies among diabetic patients had found higher rates than our study $[39,40,46]$. A study in Qatar using the same instrument as ours, i.e., DASS 21 found more than half of the diabetics have depressive, anxiety and stress symptoms [47]. The sex specific depression rates in our study were found to be within the estimated range in the general population. The differences in the rates of depression, anxiety and stress symptoms between our study and others may be attributed to differences in the screening or diagnostic instruments used, the sociocultural differences of different populations and also the sample size of the subjects. In particular, the study from Qatar had a smaller sample size of 1788.

Our study revealed that sex, ethnicity, marital status, duration of diabetes, psychiatric illness in the family and alcohol consumption were predictors of depression.
These findings are consistent with other studies which also found sex $[9,39,40,48,49]$, ethnic minority groups [50] and duration of diabetes [47] significantly associated with depression among diabetics. It is not surprising that females have a higher prevalence and risk of depression compared to males. Many factors have been implicated for this gender difference including socio-cultural and biological factors [51]. As for ethnicity, minority ethnic groups have been found to have higher depression rates as quoted in other studies [52,53]. It could be theorized that Asian Indians being the minority are more likely than Malays who form the majority to be exposed to a gamut of psychosocial stressors such as enhanced socioeconomic constraints, poor education and perceived discrimination. Consequently, these issues might augment distress thereby increasing the levels of depression among this group of minority. Similar findings and explanations were cited in a community based crosssectional study in the United States comparing depression and anxiety in respondents having insomnia and respondents without among the majority Caucasians and minority African American. In that study, Taylor et al. [54] established that African Americans were more likely to have clinically significant depression and anxiety. This was attributable to the plausible exposure to greater array of stressors in the form of discrimination, socioeconomic adversities and enhanced caregiver burden for African Americans as compared to Caucasians.

Generally, it is recognized that being married is associated with less psychiatric morbidity including depression [55-57]. Our finding of previously married and never married individuals being associated with depressive symptoms was compatible with a study by Aqbir et al. [48]. It is very likely that having a partner or spouse in a stable marriage offers emotional stability as well as shared burden in coping with challenges.

Incidentally a duration of less than 2 years of having diabetes was a predictor of depressive symptoms in this 
study. This may be attributed to inadequate or inefficient coping skills of managing diabetes by the respondents within the relatively short period since diagnosis. A related study conducted in Bahrain affirmed an association between duration of diabetes and depressive symptoms [58].

Additionally, this study elucidated the strongest predictor of depressive symptoms was having a family history or family member with psychiatric illness. It is recognized that mental illnesses in general tend to run within families [59]. In addition, in an Asian society such as ours, the responsibility of the family in caring for an ill member whether physically, emotionally or financially is very much entrenched in the culture. Hence, the caregiver's burden is occasionally translated into physical and mental health adversities such as depression [60,61].

In terms of alcohol consumption, our study showed that current drinking was an independent risk factor for depression albeit marginally. Several studies have established such an association [41,42,62].

For anxiety, our study demonstrated that having a family history of psychiatric illness was the strongest predictor. The finding is not surprising for the same reasons mentioned as for depression. That an elevated HBA1c level was an independent risk factor of anxiety in our study is also echoed by a similar association between blood glucose level and anxiety in a Mexican population [40].

With reference to current job status, this study noted that housewives and those unemployed were at risk of reporting anxiety symptoms than those who were gainfully employed. It is likely that unemployed persons lack feelings of stability and this could contribute to feelings of anxiety. However, further research is needed to show the reasons for our finding.

In this present era of modernization, anxiety has considerable influence upon the quality of life. Balancing work, family and leisure time is a challenge for both working men and women nowadays. If there was a life event preceding which could upset this delicate balance, there would definitely be an increased predilection to develop anxiety in the affected individuals. Our findings concur with several other studies conducted in this area $[63,64]$.

Physical activity has been shown to promote feelings of well being. Our findings harmonize with other studies showing association between physical inactivity and anxiety symptoms $[39,65]$. Furthermore, regular physical activity has revealed a reduction of anxiety symptoms in those who already suffer from this disorder [66].

Our data inferred that having a family member with psychiatric illness was the strongest predictor of stress. Once again caring for a family member who has a chronic illness like mental disorders is indeed a challenging task that could increase the possibilities of adversities [60,61].

Pertaining to alcohol consumption, current drinkers were strongly associated with stress symptoms. Studies have shown that drinking is used as means of coping with life's stresses [67]. Child's et al. have suggested a bidirectional relationship between alcohol use and stress [68].

Experiencing life events is inherently stressful and thus can complicate any stress level already existing in an individual [69]. Lyod et al. [70] have noted that recent severe stressors were associated with poorer glycemic control while another study found a significant correlation between stress and HbA1c [71]. Hence, our findings of life events and elevated HbA1c levels being independently associated with stress symptoms are consistent. Females were found to be at risk of stress in this study. The finding is in accordance with current literature on gender differences and stress symptoms [72-74]. We did not find any association between other sociodemographic, lifestyle and clinical factors with stress symptoms.

The strength of our study lies in its large sample size and the sampling method. However, there are also limitations which need to be considered. Firstly, this being a cross sectional study does not allow for cause and effect relationships to be studied. Secondly, the DASS 21 questionnaire is only a screening tool and not diagnostic of specific psychiatric disorders. There is also the possibility of recall biases from respondents, however this was minimized by limiting the recall period to 1 week prior to the interview using DASS 21. Information such as age, past medical history and types of medication was also verified with medical records where applicable. A point to note is that the low prevalence of family history of psychiatric illness elicited from the study could be a result of under reporting as psychiatric illness is still viewed with suspicion and stigma in our society.

As part of a long term and holistic diabetes care management, we recommend that screening for depression, anxiety and stress symptoms be conducted at regular intervals for Type II diabetics with vulnerable characteristics as mentioned above. Policies need to be in place along with appropriate intervention so that these vulnerable individuals receive optimal and timely mental health care, thus translating into better overall health outcomes.

\section{Conclusions}

The study showed that while the prevalence of depression and stress symptoms was just over $10 \%$, almost a third were classified as anxious. As these symptoms are highly correlated, they should be considered together when managing diabetic patients. Our findings could help primary 
care physicians identify high risk diabetics for screening of mental disorders. A family history of psychiatric illness was found to be a common predictor for all three symptoms. Females, current alcohol drinkers, experiencing recent life events and poor glycemic control were the other common predictors of at least two of these symptoms.

\section{Additional file}

\section{Additional file 1: DAS S 21.}

\section{Competing interests}

The authors declare that they have no competing interests.

\section{Authors' contributions}

GK was involved in the conception and design of the project, management of data collection/entry, cleaning, statistical analyses and manuscript writing/ revising. TGH assisted in the project management, data collection and manuscript writing/revising. SA was involved in the design of the project, assisted in coordination of data collection and manuscript writing/revising. ASK assisted in the project design, conducted the data collection and manuscript writing and revising. $\mathrm{KC}$ assisted in the design of the project, data management and statistical analyses. All authors read and approved the final manuscript.

\section{Acknowledgements}

The authors wish to thank the Director General of the Ministry of Health Malaysia for his permission in publishing the study findings.

\section{Author details}

${ }^{1}$ Institute for Public Health, Ministry of Health, Jalan Bangsar, Kuala Lumpur 50590, Malaysia. ${ }^{2}$ Faculty of Medicine, Universiti Teknologi MARA, Selayang Campus, Jalan Prima Selayang 7, Batu Caves, Selangor 68100, Malaysia. ${ }^{3}$ Department of Social and Preventive Medicine, University of Malaya, Kuala Lumpur 50603, Malaysia.

Received: 22 February 2013 Accepted: 22 May 2013

Published: 27 May 2013

\section{References}

1. World Health Organization (WHO): The global burden of disease: 2004 update Geneva, Switzerland: World Health Organization; 2008.

2. Institute for Public Health: The third national health and morbidity survey (NHMS III) 2006. Executive summary. Ministry of health Malaysia. Kuala Lumpur: Institute for Public Health (IPH); 2008.

3. Institute for Public Health (IPH): National health and morbidity survey 2011 (NHMS 2011). volume II: Non communicable diseases. Kuala Lumpur: Institute for Public Health (IPH); 2011.

4. Division of Burden of Disease, Institute for Public Health: Malaysian burden of disease and injury study. Health prioritization: burden of disease approach. Kuala Lumpur: Institute for Public Health (IPH), Ministry of Health Malaysia; 2006.

5. Mental health and chronic physical illnesses: The need for continued and integrated care. Vancouver: World Federation for Mental Health; 2010.

6. IDF Clinical Guidelines Task Force: Global Guideline for Type 2 diabetes. Brussels: International Diabetes Federation; 2005.

7. Coping with chronic illnesses and depression. http://www.webmd.com/ depression/guide/chronic-illnesses-depression, Accessed on 27 August 2012.

8. Goldney RD, Phillips PJ, Fisher LJ, Wilson DH: Diabetes, depression, and quality of life. A population study Diabetes Care 2004, 5(27):1066-1070.

9. Anderson RJ, Freedland KE, Clouse RE, Lustman P: The prevalence of co morbid depression in adults with diabetes: a meta-analysis. Diabetes Care 2001, 24:1069-1078.

10. Nouwen A, Winkley K, Twisk J, Lloyd CE, Peyrot M, Ismail K, Pouwer F, for the European Depression in Diabetes (EDID) Research Consortium: Type 2 diabetes mellitus as a risk factor for the onset of depression: a systematic review and meta-analysis. Diabetologia 2010, 53(12):2480-2486.
11. Kruse J, Norbert S, Wolfgang T: On the association between diabetes and mental disorders in a community sample. Diabetes Care: Results from the German National Health Interview and Examination Survey; 2003:26(6).

12. Egede LE, Zheng D, Simpon K: Comorbid depression is associated with increased health care use and expenditures in individuals with diabetes. Diabetes Care 2002, 25:464-470.

13. Finkelstein EA, Bray JW, Chen H, Larson MJ, Miller K, Tompkins C, Keme A, Manderscheid R: Prevalence and costs of major depression among elderly claimants with diabetes. Diabetes Care 2003, 26:415-420.

14. Subramaniam M, Sum CF, Pek E, Stahl D, Verma S, Liow P, Chua H, Abdin E, Chong S: Comorbid depression and increased health care utilization in individuals with diabetes. Gen Hosp Psychiatry 2009, 31(3):220-224.

15. Lustmann PJ, Anderson RJ, Freedland KE, De Groot M, Carney RM, Clouse RE: Depression and poor glycemic control:a meta-analytic review of the literature. Diabetes Care 2000, 23:934-942.

16. Lustmann PJ, Clouse RE: Depression in diabetic patients: the relationship between mood and glycemic control. J Diabetes Complications 2005, 19:113-122

17. Anderson RJ, Grigsby AB, Freedland KE, de Groot M, McGill JB, Clouse RE, Lustman PJ: Anxiety and poor glycemic control: a meta-analytic review of the literature. Int J Psychiatry Med 2002, 32(3):235-247.

18. de Groot M, Anderson R, Freedland KE, Clouse RE, Lustman PJ: Association of depression and diabetes complications: a meta-analysis. Psychosom Med 2001, 63:619-630.

19. Bruce DG, Davis WA, Starkstein SE, Davis TM: A prospective study of depression and mortality in patients with type 2 diabetes: the Fremantle diabetes study. Diabetologia 2005, 48(12):2532-2539.

20. Egede LE: Diabetes, major depression and functional disability among U. S. adults. Diabetes Care 2004, 27:421-428.

21. Schmitz N, Wang JL, Malla A, Lesage A: Joint effect of depression and chronic conditions on disability: results from a population-based study. Psychosom Med 2007, 69:332-338.

22. Ciechanowski PS, Katon WJ, Russo JE: Depression and diabetes: impact of depressive symptoms on adherence, function and costs. Arch Intern Med 2000, 160:3278-3285

23. Kilbourne AM, Reynolds CF, Good CB, Sereika SM, Justice AC, Fine MJ: How does depression influence diabetes medication adherence in older patients? Am J Geriatr Psychiatry 2005, 13(3):202-210.

24. Brown GC, Brown MM, Sharma S, Brown H, Gozum M, Denton P: Quality of life associated with diabetes mellitus in an adult population. J Diabetes Complications 2000, 14(1):18-24.

25. Das-Munshi J, Stewart R, Khalida I, Bebbington PE, Jenkins R, Prince MJ: Diabetes, common mental disorders, and disability: findings from the UK national. Surv Psychiatr Morbidity Psychosom Med 2007, 69:543-550.

26. Black SA, Markides KS, Ray LA: Depression increased incidence of adverse outcomes in older Mexican Americans with tuype 2 diabetes. Diabetes Care 2003, 26:2822-2828.

27. Zhang X, Norris SL, Gregg EW, Cheng YJ, Beckles G, Kahn HS: Depressive symptoms, and mortality among person with and without diabetes. Am J Epidemiol 2005, 161:652-660.

28. Katon WJ, Rutter C, Simon G, Lin EHB, Ludman E, Ciechanowski P, Kinder L, Young B, Korff MV: The association of comorbid depression with mortality in patients with type 2 diabetes. Diabetes Care 2004, 28:2668-2672.

29. Egede LE, Nietert P, Zheng D: Depression and all-cause and coronary heart disease mortality among adults with and without diabetes. Diabetes Care 2005, 28:1339-1345.

30. Bogner HR, Morales KH, Post EP, Bruce ML: Diabetes, depression, and death. A randomized controlled trial of a depression treatment program for older adults based in primary care (PROSPECT). Diabetes Care 2007, 30(12):3005-3010.

31. Klang Valley. http://www.freebase.com/view/en/klang_valley, Accessed on 20 January 2013.

32. Naing L, Winn T, Rusli BN: Sample size calculator for prevalence studies, Version 1.0.01. 2006. http://www.kck.usm.my/ppsg/stats_resources.htm.

33. Dupont WD, Plummer W: PS power and sample size program available for free on the Internet. Controlled Clin Trials 1997, 18:274.

34. Musa R, Fadzil MA, Zain Z: Translation, validation and psychometric properties of Bahasa Malaysia version of the Depression, Anxiety and Stress Scales (DASS). ASEAN J Psychiatr 2007, 8(2):82-89.

35. Lovibond SH, Lovibond PF: Manual for the depression anxiety stress scales. Volume 2nd Edition. Sydney: Psychology Foundation; 1995. 
36. World Health Organization: Obesity: preventing and managing the global epidemic. Report of a WHO consultation (WHO technical report series, 894). In WHO technical report series. Volume 894. Geneva: World Health Organization; 2000.

37. Weight and waist measurement: tools for adults. U.S Department of Health and Human Services, National Institute of Diabetes and Digestive and Kidney Disease; 2008.

38. WHO/IOTF/IASO: The Asia pacific perspective: redefining obesity and its treatment. Hong Kong: Health Communications Australia Pty Limited; 2000

39. Khuwaja AK, Lalani S, Dhanani R, Azam SI, Rafique G, White F: Anxiety and depression among outpatients with type 2 diabetes: a multi-centre study of prevalence and associated factors. Diabetol \& Metab Syndr 2010, 2(72):2-7.

40. Tovilla-Zárate $C$, Juárez-Rojop I, Peralta Jimenez Y, Jiménez MA, Vázquez $S$, Bermúdez-Ocaña D, Ramón-Frías T, Genis Mendoza AD, García SP, Narváez LL: Prevalence of anxiety and depression among outpatients with type 2 diabetes in the Mexican population. PLoS One 2012, 7(5):e36887.

41. Tann SS, Yabiku ST, Okamoto SK, Yanow J: TriADD: the risk for alcohol abuse, depression, and diabetes multimorbidity in the American Indian and Alaskan native population. Am Indian Alsk Native Ment Health Res 2007, 14(1):1-23.

42. Collins MM, Corcoran P, Perry IJ: Anxiety and depression symptoms in patients with diabetes. Diabet Med 2009, 26(2):153-161.

43. Dahlén I, Janson C: Anxiety and depression Are related to the outcome of emergency treatment in patients with obstructive pulmonary disease. Chest 2002, 122(5):1633-1637.

44. Bell RA, Smith SL, Arcury TA, Snively BM, Stafford JM, Quandt SA: Prevalence and correlates of depressive symptoms among rural older African Americans, native Americans, and whites with diabetes. Diabetes Care 2005, 2:823-829.

45. Sulaiman N, Hamdan A, Tamim H, Mahmood DA, Young D: The prevalence and correlates of depression and anxiety in a sample of diabetic patients in Sharjah United Arab Emirates. BMC Fam Pract 2010, 11(80). http://www.biomedcentral.com/1471-2296/11/80.

46. De Groot M, Doyle T, Hockman E, Wheeler C, Pinkerman B, Shubrook J, Gotfired R, Schwartz F: Depression among type 2 diabetes rural applachian clinic attendees. Diabetes Care 2007, 30(6):1602-1604.

47. Abdulbari B, OAA Al-Hamaq A, Dafeeah EE: High prevalence of depression, anxiety and stress symptoms among diabetes mellitus patients. Open Psychiatr J 2011, 5:5-12.

48. Agbir TM, Audu MD, Adebowale TO, Goar SG: Depression among medical outpatients with diabetes: A cross-sectional study at Jos University Teaching Hospital, Jos. Nigeria Ann Afr Med 2010, 9:5-10.

49. Nichols GA, Brown JB: Unadjusted and adjusted prevalence of diagnosed depression in type 2 diabetes. Diabetes Care 2003, 26(3):744-749.

50. Pouwer F, Geelhoed-Duijvestijn PHLM, Tack CJ, Bazelmans E, Beekman A-J, Heine RJ, Snoek FJ: Prevalence of comorbid depression is high in outpatients with Type 1 or Type 2 diabetes mellitus. Results from three outpatient clinics in the Netherlands. Diabet Med 2010, 27(2):217-224.

51. Piccinelli M, Wilkinson G: Gender differences in depression: critical review. Br J Psychiatr 2000, 177:486-492.

52. Dunlop DD, Song J, Lyons JS, Manheim LM, Chang RW: Racial/ethnic differences in rates of depression among preretirement adults. Am J Public Health 2003, 93(11):1945-1952.

53. Fisher L, Laurencin G, Chesla CA, Skaff MM, Mullan JT, Gardiner PS, Chun Kevin M: Depressive affect among four ethnic groups of male patients with type 2 diabetes. Diabetes Spectr 2004, 17(4):215-219.

54. Taylor DJ, Lichstein KL, Durrence HH, Reidel BW, Bush AJ: Epidemiology of insomnia, depression, and anxiety. Sleep 2005, 28(11):1457-1464.

55. St John PD, Montgomery PR: Marital status, partner satisfaction, and depressive symptoms in older men and women. Can J Psychiatr 2009, 54(7):487-492.

56. Afifi TO, Cox BJ, Enns MW: Mental health profiles among married, nevermarried, and separated/divorced mothers in a nationally representative sample. Soc Psychiatry Psychiatr Epidemiol 2006, 41:122-129.

57. Stutzer A, Bruno SF: Does marriage make people happy, or do happy people get married? J Socio Econ 2006, 35:326-347.

58. Almawi W, Tamim H, Al-Sayed N, Arekat MR, Al-Khateeb GM, Baqer A, Tutanji HCK: Association of comorbid depression, anxiety and stress disorders with type 2 diabetes in Bahrain, a country with a very high prevalence of type 2 diabetes. J Endocrinol Invest 2008, 31:1020-1024.
59. Barlow-Stewart K, Emery J, Metcalfe S: Psychiatric conditions. In Genetics in family medicine; the Australian handbook of general practitioners. Canberra: Biotechnology Australia, Commonwealth Department of Industry, Tourism and Resources; 2007:269-274.

60. Bookwala J, Yee JL, Schulz R: Caregiving and detrimental mental and physical health outcomes. New York: Kluwer; 2000.

61. Vanderwerker LC, Laff RE, Kadan-Lottick NS, McColl S, Prigerson HG: Psychiatric disorders and mental health service Use among caregivers of advanced cancer patients. J Clin Oncol 2005, 23(28):6899-6907.

62. Graham K, Massak A, Demers A, Rehm J: Does the association between alcohol consumption and depression depend on how they are measured? Alcohol Clin Exp Res 2007, 31(1):78-88.

63. Krantz G, Östergren PO: Double exposure: the combined impact of domestic responsibilities and job strain on common symptoms in employed Swedish women. Eur J Public Health 2001, 11:413-419.

64. Romosan F, Lenci M, Stoica I, Dehelean L: Stressful life events and anxiety disorders. Timisoara Med J 2004, 54(1):36-38

65. Hong X, Li J, Xu F, Tse LA, Liang Y, Wang Z, Yu IT, Griffiths S: Physical activity inversely associated with the presence of depression among urban adolescents in regional China. BMC Public Health 2009, 9(148). http://www.biomedcentral.com/content/pdf/1471-2458-9-148.pdf.

66. Zoeller RF: Physical activity: depression, anxiety, physical activity, and cardiovascular disease: What's the connection. Am J Lifestyle Med 2007 1(3):175-180.

67. Bressert S: Stress and drinking. Psych Central 2012. http://psychcentral.com/ lib/2006/stress-and-drinking/August, Accessed on 30 August 2012.

68. Child's E, O'Connor S, De Wit H: Bidirectional interactions between acute psychosocial stress and acute intravenous alcohol in healthy Men. Alcohol Clin Exp Res 2011, 35(10):1794-1803.

69. The truth about stress. http://www.stress-anxiety-depression.org/print/stress/ truth-about-stress.html, Accessed on 26 August 2012

70. Lloyd CE, Dyer PH, Lancashire RJ, Harris T, Daniels JE, Barnett AH: Association between stress and glycemic control in adults with type 1 (insulin-dependent) diabetes. Diabetes Care 1999, 22(8):1278-1283.

71. Kramer JR, Ledolter J, Manos GN, Bayless ML: Stress and metabolic control in diabetes mellitus: methodological issues and an illustrative analysis. Ann Behav Med 2000, 22(1):17-28.

72. The prevalence of depression, anxiety \& stress in young adults with diabetes. http://www.ads-adea.org.au/assets/ADS-ADEA/ePosters/RickieMyszka.pdf, accessed 26 August 2012.

73. Andreou E, Alexopoulos Evangelos C, Lionis C, Varvogl L, Gnardellis C, Chrousos George P, Darviri C: Perceived stress scale: reliability and validity study in Greece. Int J Environ Res Public Health 2011, 8:3287-3298.

74. Gender and Stress. http://www.apa.org/news/press/releases/stress/genderstress.aspx, Accessed on 20 January 2013.

doi:10.1186/1471-2296-14-69

Cite this article as: Kaur et al:: Depression, anxiety and stress symptoms among diabetics in Malaysia: a cross sectional study in an urban primary care setting. BMC Family Practice 2013 14:69.

\section{Submit your next manuscript to BioMed Central and take full advantage of:}

- Convenient online submission

- Thorough peer review

- No space constraints or color figure charges

- Immediate publication on acceptance

- Inclusion in PubMed, CAS, Scopus and Google Scholar

- Research which is freely available for redistribution 